\title{
Formation of Cholesteric Mesophase in Some Hydroxypropyl Cellulose-Organic Solvent Systems
}

\author{
Tetsuo TsUtsui and Ryuichi TANAKA \\ Department of Materials Science and Technology, Graduate School of Engineering Sciences, \\ Kyushu University, Hakozaki, Higashi-ku, Fukuoka 812, Japan.
}

(Received February 28, 1980)

\author{
KEY WORDS Hydroxypropyl Cellulose / Cholesteric Liquid Crystals / \\ Isotropic-Birefringent Transformation /
}

Occurrence of a cholesteric liquid-crystalline phase in concentrated aqueous solutions of hydroxypropyl cellulose (HPC) was first reported by Werbowyj and Gray in 1976, ${ }^{1}$ and the interest in this system has continued to grow since then. ${ }^{2-5}$ The iridescent colors, optical rotational dispersion spectra, ${ }^{1}$ and circular dichroism spectra ${ }^{5}$ of concentrated aqueous HPC have provided evidence for the presence of cholesteric mesophases.

In aqueous HPC solutions, however, precipitation or gelation takes place spontaneously above $40^{\circ} \mathrm{C}$ irrespective of HPC concentration, ${ }^{6}$ making the consequently complicated liquid-crystalline states of the aqueous HPC at elevated temperatures difficult to investigate. Such thermal precipitation or gelation does not occur when certain organic solvents are employed instead of water. ${ }^{6}$ Also, we recently found that HPC solution in solvents such as pyridine, ethanol, 2-methoxyethanol, and 1,4-dioxane, exhibit cholesteric liquid crystalline character.

A number of polypeptides form lyotropic cholesteric liquid crystals in appropriate organic solvents. Understanding of the behavior of these popular systems provides important clues for elucidating the properties of HPC-organic solvent systems pertaining to the cholesteric liquid-crystalline structures of these systems.

The HPC used in this study was of commercial reagent and supplied by Tokyo Kasei Kogyo Co. Ltd., Japan. The average number of propylene oxide units per anhydroglucose unit, MS, was stated to be around 3.5. The molecular weight of the HPC was $8.7 \times 10^{4}$, as determined by solution viscometry in an ethanol-water mixture. ${ }^{7}$ Pyridine, ethanol, 2methoxyethanol, and 1,4-dioxane were commercial reagents and used without further purification.

For the microscopic observation of liquidcrystalline states, a $50 \%$ (wt/wt) HPC solution was prepared for each solvent in a small screw vial, mixed homogeneously, allowed to stand overnight, and then loaded into a flat glass cell in $0.15 \mathrm{~mm}$ in thickness. The sample cells were observed by a Nikon polarizing optical microscope, under crossed polarizers at room temperature. After the sample cells were allowed to stand at room temperature for a few hours, regular retardation lines, characteristic of cholesteric liquid-crystalline states, became visible in both the HPC-pyridine and HPC-2-methoxyethanol systems. Figure 1 shows the cholesteric liquid-crystalline state of the $50 \%$ HPC-pyridine solution. The cholesteric pitches of

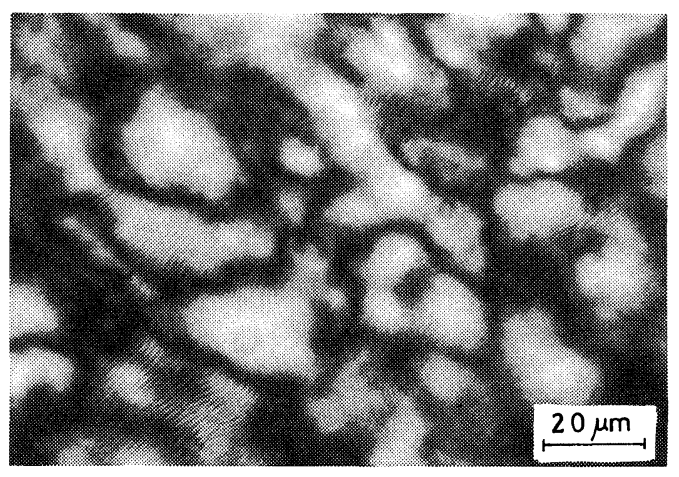

Figure 1. Polarizing micrograph of a $50 \%$ (wt/wt) HPC-pyridine solution, viewed under crossed polarizers. 
the $50 \%$ HPC-pyridine and the $50 \%$ HPC-2methoxyethanol were 2.5 and $1.8 \mu \mathrm{m}$, respectively. On the other hand, the cholesteric pitches of the ethanol and the 1,4-dioxane solutions were too small to be observed with the optical microscope. The cholesteric pitches of the aqueous HPC systems have been reported to be much smaller than the above values; these pitches are comparable with the wavelength of visible light.

In order to determine the critical concentrations below which the solutions no longer exhibit a liquidcrystalline character, we prepared and observed HPC solutions of several different concentrations in the range $40-65 \%(\mathrm{wt} / \mathrm{wt})$ for each solvent. HPC and the solvents were weighed into $10-\mathrm{mm}$ glass tubes, degased, sealed, and allowed to stand for a few days at $60^{\circ} \mathrm{C}$ with the tops and bottoms inverted intermittently to assist homogeneous mixing. While all the $40 \%$ solutions were transparent and isotropic, the solutions with higher HPC contents were birefringent. All the solutions were classified as either being perfectly isotropic or being entirely birefringent. No bi-phasic solutions were encountered even after the solutions were kept at room temperature for as long as three months. Instead, every birefringent solution became transparent within a $5^{\circ} \mathrm{C}$ temperature range, if heated slowly. The reverse transformation also occurred within the same temperature range when the hot solution was allowed to cool. In Figure 2, the temperature of the

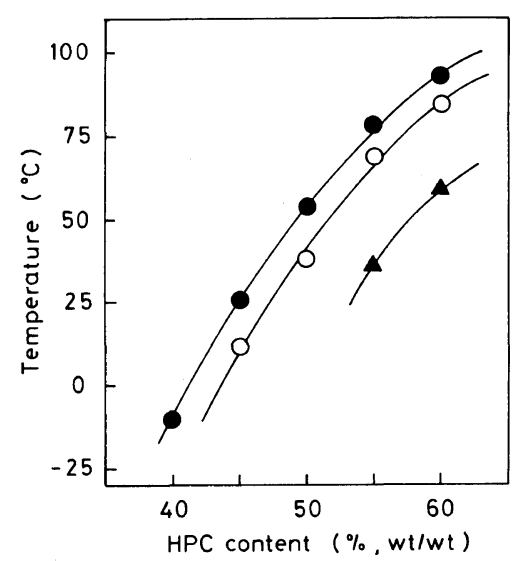

Figure 2. Isotropic to birefringent transformation temperature plotted against HPC concentration. Solvent: pyridine; $\bigcirc$, 2-methoxyethanol; $\boldsymbol{\Delta}$, ethanol. isotropic to birefringent transformation is plotted against HPC concentration.

The absence of a bi-phasic region in the temperature-concentration diagram and the presence of a well-defined isotropic to birefringent transformation are reminiscent of the "induced coil to helix transition" reported for several polypeptide solutions. For example, poly( $\gamma$-benzyl L-glutamate) molecules are random coils in a dilute dichloroacetic-acid solution. When the solution becomes concentrated, the random coils transform into helices at a certain critical polymer concentration, and simultaneously, the isotropic solution turns into anisotropic liquid crystals. ${ }^{8}$ Similar phenomena have been observed by Iizuka and his coworkers on aqueous solutions of poly(sodium Lglutamate) and poly(L-lysine hydrobromide) ${ }^{9,10}$ Although the random coil to rigid chain transition has not been mentioned in the literature for any HPC solutions, we are tempted to postulate that the reversible isotropic to birefringent transformations found in the HPC-organic solvent systems have some bearing on the change in flexibility of HPC molecules in response to temperature variation. ${ }^{11}$

\section{REFERENCES}

1. R. S. Werbowyj and D. G. Gray, Mol. Cryst. Liq. Cryst. Lett., 34, 97 (1976).

2. R. S. Werbowyj and D. G. Gray, Polym. Prepr., Am. Chem. Soc., Div. Polym. Chem., 20, No. 1, 102 (1979).

3. Y. Onogi, J. L. White, and J. F. Fellers, Polym. Prepr., Am. Chem. Soc., Div. Polym. Chem., 20, No. 1, 57 (1979).

4. T. Asada, M. Yoshida, and S. Onogi, Polym. Prepr., Jpn., 27, No. 6, 1366 (1978).

5. H. Toriumi, K. Miyasaka, and I. Uematsu, Polym. Prepr., Jpn., 28, No. 2, 363 (1979).

6. E. D. Klug, J. Polym. Sci., C, 36, 491 (1971).

7. M. G. Wirick and M. H. Woldman, J. Appl. Polym. Sci., 14, 579 (1970).

8. S. Ya. Frenkel', L. G. Shaltyko, and G. K. Elyashevich, J. Polym. Sci., C, 30, 47 (1970).

9. E. Iizuka, Y. Kondo, and Y. Ukai, Polym. J., 9, 135 (1977).

10. Y. Kondo, Y. Ukai, and E. Iizuka, Polym. J., 10, 631 (1978).

11. P. Pincus and P. G. de Gennes, J. Polym. Sci., Polym. Symp., No. 65, 85 (1978). 